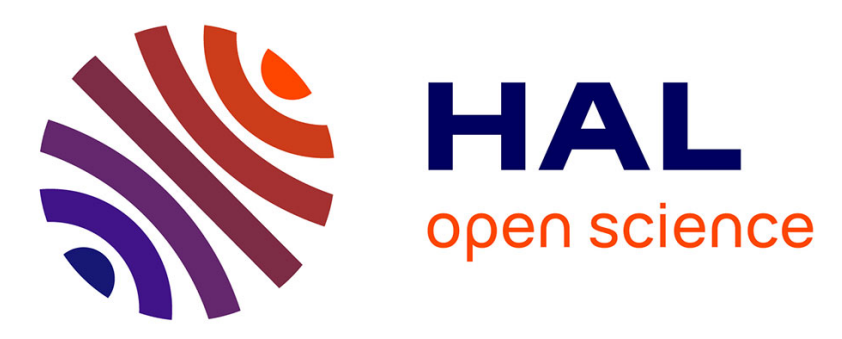

\title{
General equilibrium analysis in ordered topological vector spaces
}

\author{
Charalambos D. Aliprantis, Monique Florenzano, Rabee Tourky
}

\section{To cite this version:}

Charalambos D. Aliprantis, Monique Florenzano, Rabee Tourky. General equilibrium analysis in ordered topological vector spaces. Journal of Mathematical Economics, 2004, 40 (3-4), pp.247-269. 10.1016/j.mateco.2003.11.004 . halshs-00086791

\section{HAL Id: halshs-00086791 https://shs.hal.science/halshs-00086791}

Submitted on 19 Jul 2006

HAL is a multi-disciplinary open access archive for the deposit and dissemination of scientific research documents, whether they are published or not. The documents may come from teaching and research institutions in France or abroad, or from public or private research centers.
L'archive ouverte pluridisciplinaire HAL, est destinée au dépôt et à la diffusion de documents scientifiques de niveau recherche, publiés ou non, émanant des établissements d'enseignement et de recherche français ou étrangers, des laboratoires publics ou privés. 


\title{
GENERAL EQUILIBRIUM ANALYSIS IN ORDERED TOPOLOGICAL VECTOR SPACES*
}

\author{
CHARALAMBOS D. ALIPRANTIS, ${ }^{1}$ MONIQUE FLORENZANO, ${ }^{2}$ AND RABEE TOURKY ${ }^{3}$
}

1 Department of Economics, Krannert School of Management, Purdue University, 403 West State Street, W. Lafayette, IN 47907-2056, USA; aliprantis@mgmt.purdue.edu

2 CNRS-CERMSEM, MSE Université Paris 1, 106-112 boulevard de l'Hôpital, 75647 Paris Cedex 13, FRANCE; monique.florenzano@univ-paris1.fr

3 Department of Economics and Commerce, University of Melbourne, Parkville 3052, Melbourne, AUSTRALIA; rtourky@unimelb.edu.au

\begin{abstract}
The second welfare theorem and the core-equivalence theorem have been proved to be fundamental tools for obtaining equilibrium existence theorems, especially in an infinite dimensional setting. For well-behaved exchange economies that we call proper economies, this paper gives (minimal) conditions for supporting with prices Pareto optimal allocations and decentralizing Edgeworth equilibrium allocations as non-trivial quasi-equilibria. As we assume neither transitivity nor monotonicity on the preferences of consumers, most of the existing equilibrium existence results are a consequence of our results. A natural application is in Finance, where our conditions lead to new equilibrium existence results, and also explain why some financial economies fail to have equilibria.
\end{abstract}

KEYwords: Equilibrium, valuation equilibrium, Pareto optimum, Edgeworth equilibrium, properness, ordered topological vector space, Riesz-Kantorovich formula, sup-convolution

JEL Classification: C61, C65, D41, D51, G11, G12.

*This version of the paper was prepared during visits of C. D. Aliprantis to CERMSEM, of C. D. Aliprantis and M. Florenzano to the Economic Theory Center of the University of Melbourne, and of M. Florenzano and R. Tourky to Purdue University. The hospitality of these institutions is gratefully acknowledged. Presented at Lima (Peru) during the V Seminario International de Optimisación y Areas affines (October 2001), at Santiago de Chile during the VIth Franco-Chilian Conference in Applied Mathematics (December 2001), at the University of Warwick, at Salamanca during the XVII Simposio de Análisis Económico (December 2002), at Bielefeld during the 12th European Workshop on General Equilibrium Theory (May 2003), the paper has benefitted of the comments of these diverse audiences. We thank O. Cornejo and F. Martins da Rocha for valuable conversations. The research of C. D. Aliprantis is supported by the NSF Grants EIA-0075506 and SES-0128039. The research of R. Tourky is funded by the Australian Research Council Grant A00103450. 


\section{INTRODUCTION}

The welfare theorems and the core-equivalence theorem state important optimal properties of general equilibrium allocations. Loosely speaking, given the technological and resource limitations, individual needs and tastes that define an economy, every equilibrium allocation is as well a Pareto optimal allocation, a core allocation and an Edgeworth equilibrium. Converse results give conditions under which every Pareto optimal allocation can be supported by a non-zero price linear functional such that each consumer minimizes her expenditure and each firm maximizes its profit while every Edgeworth equilibrium is a quasi-equilibrium allocation when associated with an appropriate non-zero price linear functional. These converse results have been proved to be the fundamental tools for obtaining equilibrium existence theorems. Directly or indirectly, their validity in an infinite dimensional setting generally relies either, as in [18], on an interiority property of the positive cone of the commodity space (assumed to be an ordered topological vector space) or on the so-called properness assumptions made on the characteristics of the economy. In the latter case, the lattice structure of the commodity space plays an essential role in establishing decentralization results.

After the seminal paper of Mas-Colell [28] in 1986, the lattice requirements have been weakened and it is now well-known that for an equilibrium existence theorem, the topology of the vector lattice commodity space need not be locally solid. However, in [20], [24], [29], [30], [35], [39], the commodity space is still assumed to be a vector lattice and the lattice properties are used in the proofs. The aim of this paper is to look at what extent the lattice structure itself of the commodity space can be given up. More precisely, its aim is to state the (minimal) properties that have to be assumed on the order structure of the commodity space for guaranteeing the validity of the decentralization theorems.

A first attempt was done in Aliprantis-Tourky-Yannelis [11] where the characterization of Pareto optimality and Edgeworth equilibrium is given using non-linear prices for decentralizing the relevant allocations. This paper goes further and looks for decentralization with linear prices. We deal with well-behaved exchange economies that we call proper economies. Such economies, defined on a locally convex Hausdorff ordered topological vector space with topologically bounded order intervals, have consumption sets equal to the positive orthant, (semi-) continuous or concave preferences, preferred sets extendable to the entire space as convex sets with a nonempty interior, and the positive total endowment as a common properness direction. We show that necessary and/or sufficient conditions for supporting with continuous linear functionals weakly Pareto optimal allocations and decentralizing with continuous linear prices Edgeworth equilibria of any well-behaved exchange economy can be expressed in terms of the properties of the Riesz-Kantorovich formula associated with a finite list of linear functionals on the commodity space. These properties should be understood as a condition of compatibility between the topology and the order structure of the commodity space. They are implied by the assumption currently made that the commodity space is a vector lattice equipped with a locally convex topology whose positive cone is closed and the topological dual is a vector sublattice of the order dual. They are a-fortiori implied by the assumption that the commodity space is a topological vector lattice, so that we get as consequence of our main results most of earlier results for an exchange economy.

At this point, it is worth recalling that weakly Pareto optimal allocations and Edgeworth equilibria exist under mild conditions on well-behaved economies (for statements and proofs in an infinite dimensional setting, see for example [3], [11], [22], [28]; see also [12] for a proof of the existence of Edgeworth equilibria in economies with non-compact attainable sets). Their existence does not 
require any lattice property of the commodity space. Hence, revisiting the main results of welfare economics in infinite dimensional spaces, we actually establish the conditions of possibility of the general equilibrium theory in ordered topological vector spaces.

Our paper is organized as follows. In section 2, we recall the notion of the Riesz-Kantorovich functional associated with a finite list of (linear) functionals, a notion that we put in relation with the concept of sup-convolution, well-known in optimization. In section 3 , we define the model and posit assumptions as well on the commodity space as on the characteristics of the economy. Let us emphasize here that we do not assume any kind of monotonicity or transitivity on the preferences of consumers. Sections 4 and 5 are devoted to our main results where we give conditions for supporting Pareto optimal allocations and decentralizing Edgeworth equilibria with continuous linear prices. In the last section, we indicate some consequences of these results regarding the equilibrium existence problem. Most of the currently used commodity spaces are topological vector lattices or at least vector lattices with a lattice ordered topological dual. Section 6 is devoted to examples of commodity spaces that do not satisfy these assumptions and thus are not covered by existing results (e.g. Mas-Colell [28], Mas-Colell-Richard [30], among others). Equilibrium models in Finance are the most convincing examples where the commodity (portfolio) space, after re-ordering by the portfolio dominance ordering introduced in [4], need not be a lattice. We explain why some models fail to have an equilibrium. We also exhibit finite dimensional and infinite dimensional settings that lead to equilibrium existence results not covered by any available theorem. The last example is also of economic interest. The commodity space used by HindyHuang [25] for modeling intertemporal preferences for uncertain consumption in continuous time is a vector lattice but its topological dual is not a lattice. In such a setting, we indicate how to recover equilibrium existence.

\section{MAThematical PRELIMINARIES}

For details regarding Riesz spaces that are not explained below we refer the reader to [5] and [6]. This paper will utilize the notion of the Riesz-Kantorovich formula that was introduced in [10] and used extensively in [11]. We shall briefly introduce this formula here and refer the reader to [9] for a complete discussion regarding the Riesz-Kantorovich formula.

We start with the following classical result from the theory of partially ordered vector spaces due to F. Riesz and L. V. Kantorovich.

Theorem 2.1 (Riesz-Kantorovich). If $L$ is an ordered vector space with a generating cone and the Riesz Decomposition Property, ${ }^{1}$ then the order dual $L^{\sim}$ is a Riesz space and for each $f, g \in L^{\sim}$ and $x \in L_{+}$its lattice operations are given by:

(1) $\quad[f \vee g](x)=\sup \left\{f(y)+g(z): y, z \in L_{+}\right.$and $\left.y+z=x\right\}$

(2) $[f \wedge g](x)=\inf \left\{f(y)+g(z): y, z \in L_{+}\right.$and $\left.y+z=x\right\}$.

In particular, note that if $L$ has the Riesz Decomposition Property, then for any finite collection of linear functionals $f_{1}, f_{2}, \ldots, f_{m} \in L^{\sim}$ their supremum in $L^{\sim}$ at each $x \in L_{+}$is given by

$$
\left[\bigvee_{i=1}^{m} f_{i}\right](x)=\sup \left\{\sum_{i=1}^{m} f_{i}\left(x_{i}\right): x_{i} \in L_{+} \text {for each } i \text { and } \sum_{i=1}^{m} x_{i}=x\right\}
$$

\footnotetext{
${ }^{1}$ The Decomposition Property states that if $x, y_{1}, y_{2} \in L_{+}$satisfy $0 \leq x \leq y_{1}+y_{2}$, then there exist elements $x_{1}$ and $x_{2}$ such that $0 \leq x_{1} \leq y_{1}, 0 \leq x_{2} \leq y_{2}$ and $x=x_{1}+x_{2}$. The order dual $L \sim$ is the vector space consisting of all linear functionals on $L$ which map order intervals of $L$ to order bounded subsets of $\mathbb{R}$, ordered by the relation $f \geq g$ whenever $f(x) \geq g(x)$ for all $x \in L_{+}$.
} 
For any positive integer $m$ and $x \in L_{+}$define

$$
\mathcal{A}_{x}^{m}=\left\{\left(x_{1}, \ldots, x_{m}\right) \in L_{+}^{m}: \sum_{i=1}^{m} x_{i}=x\right\} .
$$

The formula $(\star)$ that gives the supremum of the order bounded linear functionals $f_{1}, \ldots, f_{m}$ is called the Riesz-Kantorovich formula of these functionals. The useful observation here is that if each $f_{i}$ is an arbitrary function from $L_{+}$to $(-\infty, \infty]$, then the right-hand side of $(\star)$ still defines an extended real number for each $x \in L_{+}$. That is, the formula appearing in $(\star)$ defines a function from $L_{+}$to $(-\infty, \infty]$ called the Riesz-Kantorovich functional of the $m$-tuple of functions $f=\left(f_{1}, \ldots, f_{m}\right)$ and denoted $\mathcal{R}_{f}$. In other words, $\mathcal{R}_{f}: L_{+} \rightarrow(-\infty, \infty]$ is defined by

$$
\mathcal{R}_{f}(x):=\sup \left\{\sum_{i=1}^{m} f_{i}\left(x_{i}\right):\left(x_{1}, \ldots, x_{m}\right) \in \mathcal{A}_{x}^{m}\right\}
$$

for each $x \in L_{+}$.

If each $f_{i}$ is a function that carries order intervals to bounded subsets of $\mathbb{R}$, then the RieszKantorovich functional is real-valued. Moreover, if each $f_{i}$ is super-additive and positively homogeneous, then the Riesz-Kantorovich functional $\mathcal{R}_{f}$ is also super-additive and positively homogeneous; in particular, it is a concave function. Using as prices the Riesz-Kantorovich functional of a list of personalized prices, a new theory of value was presented in [11].

Let us now associate with each $f_{i}: L_{+} \rightarrow(-\infty, \infty)$ the function $\widehat{f_{i}}: L \rightarrow[-\infty, \infty)$ defined by

$$
\widehat{f}_{i}(x):= \begin{cases}f_{i}(x) & \text { if } x \in L_{+} \\ -\infty & \text { otherwise }\end{cases}
$$

If each $f_{i}$ is finite-valued, it is easy to recognize in $\mathcal{R}_{f}(x)$ for $x \in L_{+}$the value at $x$ of the sup-convolution ${ }^{2}$ of functions $\widehat{f_{i}}$ defined by

$$
\left[\nabla_{i=1}^{m} \widehat{f}_{i}\right](x):=\sup \left\{\sum_{i=1}^{m} \widehat{f}_{i}\left(x_{i}\right): \sum_{i=1}^{m} x_{i}=x\right\} .
$$

Definition 2.2. We will say that $\mathcal{R}_{f}$ is exact at $x$ with respect to $\left(x_{1}, \ldots, m\right) \in E_{+}^{m}$ such that $x=\sum_{i=1}^{m} x_{i}$ if the sup-convolution is exact at $x$ with respect to $\left(x_{1}, \ldots, x_{m}\right) \in E_{+}^{m}$, that is, if

$$
\mathcal{R}_{f}(x)=\left(\nabla_{i=1}^{m} \widehat{f}_{i}\right)(x)=\sum_{i=1}^{m} \widehat{f}_{i}\left(x_{i}\right)=\sum_{i=1}^{m} f_{i}\left(x_{i}\right) .
$$

Let $\left\langle X, X^{\prime}\right\rangle$ be a dual system and let $f \in \overline{\mathbb{R}}^{X}$. Recall that $y \in X^{\prime}$ is a supergradient of $f$ at $x$ if $f(x)$ is finite and if $f\left(x^{\prime}\right)-f(x) \leq\left\langle x^{\prime}-x, y\right\rangle$ for all $x^{\prime} \in X$. The (eventually empty) set of all supergradients of $f$ at $x$ is called the superdifferential at $x$ of the function $f$ and denoted $\partial f(x)$. The following result (see for example [27, Proposition 6.6.4]) will be used in our work extensively.

\footnotetext{
${ }^{2}$ Let $f \in \overline{\mathbb{R}}^{L}$ and $g \in \overline{\mathbb{R}}^{L}$ be extended real-valued functions. Using the convention $+\infty+(-\infty)=-\infty+(+\infty)=$ $-\infty$, the formula $[f \nabla g](x)=\sup \{f(y)+g(z): y, z \in L$ and $y+z=x\}$ defines an extended real-valued function $f \nabla g$ called the sup-convolution of $f$ and $g$. The expression $f \nabla g$ is also called by Rockafellar and Wets [37] the hypoaddition of functions $f$ and $g$, because if hypo $f$ denotes the hypograph of $f$, one has hypo $(f \nabla g)=$ hypo $f+$ hypo $g$, as long as the supremum defining $[f \nabla g](x)$ is attained when finite.
} 
Theorem 2.3 (Moreau [32]). Assume that $\left\langle X, X^{\prime}\right\rangle$ is an arbitrary dual system. For each $i=$ $1, \ldots, m$ let $g_{i}: X \rightarrow[-\infty, \infty]$ be a non identically equal to $-\infty$ function. If the sup-convolution $\nabla_{i=1}^{m} g_{i}$ is exact at $x$ with respect to $\left(x_{1}, \ldots, x_{m}\right)$ that satisfies $x=\sum_{i=1}^{m} x_{i}$, then

$$
\partial\left[\nabla_{i=1}^{m} g_{i}\right](x)=\bigcap_{i=1}^{m} \partial g_{i}\left(x_{i}\right) .
$$

\section{THE ECONOMIC MODEL}

We shall deal with pure exchange economies. The commodity space is an ordered linear vector space $L$ equipped with a Hausdorff locally convex topology $\tau$ such that:

A1: The positive cone $L_{+}$of $L$ is generating (i.e., $L=L_{+}-L_{+}$) and $\tau$-closed.

A2: The order intervals of $L$ are $\tau$-bounded.

In what follows, for the sake of notational convenience, $M^{+}$or $M_{+}$will denote the positive cone of an ordered linear space $M$. The topological dual of $(L, \tau)$ (i.e., the vector space of all $\tau$-continuous linear functionals on $L$ ) will be denoted $L^{\prime}$. The algebraic dual of $L$ (i.e., the vector space of all linear functionals on $L$ ) is denoted $L^{*}$. The order dual of $L$ (i.e., the vector space of all orderbounded linear functionals on $L$ ) is denoted $L^{\sim}$. Since every order interval of $L$ is $\tau$-bounded, it follows that $L^{\prime} \subseteq L^{\sim} \subseteq L^{*}$.

If $L$ is a vector lattice, the assumptions $\mathbf{A} \mathbf{1}$ and $\mathbf{A} \mathbf{2}$ are satisfied by the following ones used by Mas-Colell-Richard [30] and many others:

B1: The positive cone $L_{+}$of $L$ is $\tau$-closed; and

B2: $L^{\prime}$ is a vector sublattice of $L^{\sim}$.

Both sets of assumptions are implied by Mas-Colell's assumption in [28] and [29] that $L$ is a topological vector lattice; see [5, Theorem 5.7].

On $L$ as commodity space, let us consider an economy

$$
\mathcal{E}=\left(X_{i}, P_{i}, \omega_{i}\right)_{i \in I},
$$

where $I=\{1, \ldots, m\}$ is a finite set of $m$ consumers. Each consumer $i \in I$ is characterized by a consumption set $X_{i} \subseteq L$, an initial endowment $\omega_{i}$ and an irreflexive preference correspondence $P_{i}: X_{i} \rightarrow X_{i}$ (i.e., $x_{i} \notin P_{i}\left(x_{i}\right)$ for each $\left.x_{i} \in X_{i}\right)$.

Let $\omega=\sum_{i \in I} \omega_{i}$ be the total endowment, and let $\mathcal{A}_{\omega}$ be the set of all (feasible or attainable) allocations of $\mathcal{E}$, that is,

$$
\mathcal{A}_{\omega}=\left\{x=\left(x_{i}\right)_{i \in I} \in \prod_{i \in I} X_{i}: \sum_{i \in I} x_{i}=\omega\right\} .
$$

The following notions of equilibrium and optimality for $\mathcal{E}$ are standard.

Definition 3.1. A pair $(x, p)$ consisting of an allocation $x$ and a non-zero linear functional $p$ is said to be:

(1) a quasi-valuation equilibrium, if for every $i \in I, y_{i} \in P_{i}\left(x_{i}\right)$ implies $p\left(y_{i}\right) \geq p\left(x_{i}\right)$,

(2) a quasi-equilibrium, if it is a quasi-valuation equilibrium and for every $i \in I$ we have $p\left(x_{i}\right)=p\left(\omega_{i}\right)$

(3) a valuation equilibrium (resp. an equilibrium), if it is a quasi-valuation equilibrium (resp. a quasi-equilibrium) and if $y_{i} \in P_{i}\left(x_{i}\right)$ actually implies $p\left(y_{i}\right)>p\left(x_{i}\right)$. 
Definition 3.2. A quasi-valuation equilibrium (resp. a quasi-equilibrium) $(x, p)$ is said to be nontrivial, if for some $i \in I$ we have

$$
\inf \left\{p\left(z_{i}\right): z_{i} \in X_{i}\right\}<p\left(x_{i}\right)\left(\text { resp. } \inf \left\{p\left(z_{i}\right): z_{i} \in X_{i}\right\}<p\left(\omega_{i}\right)\right) .
$$

In this paper, we will be interested only in nontrivial quasi-valuation equilibria or nontrivial quasi-equilibria. ${ }^{3}$ We now introduce some special properties of feasible allocations.

Definition 3.3. A (feasible) allocation $x$ is said to be:

(1) individually rational, if $\omega_{i} \notin P_{i}\left(x_{i}\right)$ for each $i \in I$,

(2) weakly Pareto optimal, if there is no allocation $y$ satisfying $y_{i} \in P\left(x_{i}\right)$ for each $i \in I$,

(3) a core allocation, if it cannot be blocked by any coalition in the sense that there is no coalition $S \subseteq I$ and some $y \in \prod_{i \in S} X_{i}$ such that:

(a) $\sum_{i \in S} y_{i}=\sum_{i \in S} \omega_{i}$, and

(b) $y_{i} \in P_{i}\left(x_{i}\right)$ for all $i \in S$,

(4) an Edgeworth equilibrium, if for every integer $r \geq 1$ the $r$-fold replica of $x$ belongs to the core of the $r$-fold replica of the economy $\mathcal{E} .^{4}$

As proved in Florenzano [22], if preferred sets are convex and open in convex consumption sets containing the individual endowments, an Edgeworth equilibrium is a feasible allocation $x$ such that there exist no $\tau=\left(\tau_{i}\right)_{i \in I} \in[0,1]^{I} \backslash\{0\}$ and $y \in \prod_{i \in I} X_{i}$ such that:

(i) $\sum_{i \in I} \tau_{i} y_{i}=\sum_{i \in I} \tau_{i} \omega_{i}$, and

(ii) $y_{i} \in P_{i}\left(x_{i}\right)$ for all $i \in S$ with $\tau_{i}>0$.

The same holds true without any continuity condition on the preferred sets that are defined in convex consumption sets by concave utility functions.

It easily follows from the previous definitions that every equilibrium allocation is an Edgeworth equilibrium and, consequently, a core allocation and a weakly Pareto optimal and individually rational allocation. In order to get converse statements, we will employ in this paper two notions of properness. The first one corresponds to the one defined by Mas-Colell [28] for complete preorderings.

Definition 3.4. Let $v \in L_{+}$be such that $v>0$.

(1) A correspondence $P: L_{+} \rightarrow L_{+}$is said to be pointwise v-proper at $x \in L_{+}$, if there is an open convex cone $\Gamma_{x}$ with vertex $x$ such that $x-v \in \Gamma_{x}$ and $P(x) \cap \Gamma_{x}=\varnothing$. A correspondence $P: L_{+} \rightarrow L_{+}$is pointwise proper at $x \in L_{+}$, if there is some $v>0$ in $L_{+}$such that $P$ is pointwise $v$-proper at $x$.

(2) A utility function $u: L_{+} \rightarrow \mathbb{R}$ is said to be pointwise $v$-proper at $x \in L_{+}$, if the correspondence defined by $P(z):=\left\{y \in L_{+}: u(y)>u(z)\right\}$ is pointwise $v$-proper at $x$. The function $u$ is pointwise proper at $x \in L_{+}$, if there is some $v>0$ such that $u$ is pointwise $v$-proper at $x$.

\footnotetext{
${ }^{3}$ If $(x, p)$ is some trivial quasi-valuation equilibrium (resp. some trivial quasi-equilibrium), then for every allocation $y$, the pair $(y, p)$ is also a quasi-valuation equilibrium (resp. a quasi-equilibrium). If the quasi-valuation equilibrium (resp. quasi-equilibrium) $(x, p)$ is nontrivial, then it is well-known that (under some additional continuity condition on preferences or concavity for utility functions, and some irreducibility assumption on the economy) $(x, p)$ is actually a valuation equilibrium (resp. an equilibrium).

${ }^{4}$ The ideas in this definition go back to Debreu-Scarf [19]. An important reference is also [15]. Edgeworth equilibria were first introduced and studied in [2].
} 
As remarked by Mas-Colell, the pointwise $v$-properness at $x \in \overline{P(x)}$ is a necessary and sufficient condition for the preferred set $P(x)$ to be supported at $x$ by a continuous linear functional $f$ such that $f(v)>0$. If $u: L_{+} \rightarrow \mathbb{R}$ is concave, the pointwise $v$-properness of the utility function $u$ at $x \in \operatorname{cl}\left\{y \in L_{+}: u(y)>u(x)\right\}$ is a necessary and sufficient condition for $\widehat{u}$ to be superdifferentiable at $x .^{5}$

The second notion of properness, introduced by Tourky [39], is a stronger one, at least when properness is assumed at $x \in \operatorname{cl} P(x)$.

Definition 3.5. Let $v \in L_{+}$be such that $v>0$.

(1) A correspondence $P: L_{+} \rightarrow L_{+}$is v-proper at $x \in L_{+}$, if there is a convex set $\widehat{P}(x)$ such that $x+v$ is a $\tau$-interior point of $\widehat{P}(x)$ and $\widehat{P}(x) \cap L_{+}=P(x)$. The correspondence $P: L_{+} \rightarrow L_{+}$is proper at $x \in L_{+}$, if there is some $v>0$ in $L_{+}$such that $P$ is v-proper at $x .^{6}$

(2) A utility function $u: L_{+} \rightarrow \mathbb{R}$ is said to be v-proper at $x \in L_{+}$, if the correspondence defined by $P(z):=\left\{y \in L_{+}: u(y)>u(z)\right\}$ is v-proper at $x$. The function $u$ is proper at $x \in L_{+}$, if there is some $v$ such that $u$ is $v$-proper at $x$.

We now define the notion of a proper economy.

Definition 3.6. An exchange economy $\mathcal{E}=\left(X_{i}, P_{i}, \omega_{i}\right)_{i \in I}$ will be referred to as an $\omega$-proper economy, if it satisfies the following properties:

(1) $X_{i}=L_{+}$and $\omega_{i} \in L_{+}$for each consumer $i$, and $\omega=\sum_{i \in I} \omega_{i}>0$,

(2) for each $i \in I$ and every weakly Pareto optimal allocation $x=\left(x_{i}\right)_{i \in I}$ we have $x_{i} \in \operatorname{cl} P_{i}\left(x_{i}\right)$ and:

(a) $P_{i}\left(x_{i}\right)$ is $\tau$-open in $L_{+}$or $P_{i}\left(x_{i}\right)=\left\{y_{i} \in L_{+}: u_{i}\left(y_{i}\right)>u\left(x_{i}\right)\right\}$ for some concave utility function $u_{i}: L_{+} \rightarrow \mathbb{R}$

(b) there is a convex set $\widehat{P}_{i}\left(x_{i}\right)$ such that the vector $x_{i}+\omega$ is a $\tau$-interior point of $\widehat{P}_{i}\left(x_{i}\right)$ and $\widehat{P}_{i}\left(x_{i}\right) \cap L_{+}=P_{i}\left(x_{i}\right)$.

The following result will be useful in our work here.

Proposition 3.1. In an $\omega$-proper economy, assume that $x$ is a weakly Pareto optimal allocation and that, for some $p \in L^{\prime},(x, p)$ is a quasi-valuation equilibrium (resp. a quasi-equilibrium). Then $(x, p)$ is nontrivial if and only if $p(\omega)>0$.

Proof. Assume that $x=\left(x_{1}, \ldots, x_{m}\right)$ is a weakly Pareto optimal allocation and that $(x, p)$, with $p \in L^{\prime}$, is a quasi-valuation equilibrium (resp. a quasi-equilibrium) for an $\omega$-proper economy with $m$ consumers and total endowment $\omega$.

If the quasi-valuation equilibrium (resp. quasi-equilibrium) $(x, p)$ is nontrivial, then, in view of properties (a) and (b) in part (2) of Definition 3.6, for some $i$, we have $p\left(x_{i}+\omega\right)>p\left(x_{i}\right)$. This implies that $p(\omega)>0$.

\footnotetext{
${ }^{5}$ The existence of Lagrange multipliers for a convex programming problem will be used at several instances in this paper. A simple proof can be found in Barbu and Precupanu [16, Chapter 3, Theorem 1.1]. See also [21] and [1, Chapter 5, Theorem 5.77].

${ }^{6}$ For complete preorderings on $L_{+}$, Mas-Colell's [28] uniform properness is basically equivalent to the pointwise properness at every $x \in L_{+}$with a uniform properness vector $v$ and a uniform open convex cone $\Gamma_{x}-\{x\}$, with vertex 0 containing $-v$. As shown in [39], when preferences are complete preorderings, Mas-Colell's uniform properness is strictly stronger than properness at $x \in L_{+}$. For related properness conditions on preferences, see Yannelis and Zame [40], Araujo and Monteiro [14], Podczeck [35], Deghdak and Florenzano [20], Florenzano and Marakulin [24].
} 
For the converse, assume that $p(\omega)>0$. The ordered subspace

$$
L_{\omega}=\bigcup_{\lambda>0} \lambda[-\omega, \omega]
$$

is Archimedean (a property inherited from $L$ of which the positive cone is closed) and has $\omega$ as an order unit. ${ }^{7}$ Its order topology (i.e., the finest locally convex topology on $L_{\omega}$ for which every order interval is bounded) is normable (see Schaefer [38, Chapter V 6.2]). More precisely, the gauge $p_{\omega}$ of $[-\omega, \omega]$, defined by

$$
\|z\|_{\omega}=\inf \{\lambda>0: \quad-\lambda \omega \leq z \leq \lambda \omega\},
$$

is a norm on $L_{\omega}$, so that for the order topology, $\omega$ is an interior point of $L_{\omega}^{+}$. The same holds obviously true for $\frac{1}{m} \omega$.

Assume now that for every $i$ and for every $z \in L_{\omega}^{+}$we have $p(z) \geq p\left(x_{i}\right)$. Fix $z \in[-\omega, \omega]$ and then choose $\varepsilon>0$ such that $\frac{1}{m} \omega+\varepsilon z \in L_{\omega}^{+}$. Then for every $i$ we have

$$
p\left(\frac{1}{m} \omega+\varepsilon z\right) \geq p\left(x_{i}\right) .
$$

Summing over $i$, we get $m \varepsilon p(z) \geq 0$. So, $p(z) \geq 0$ for all $z \in[-\omega, \omega]$, and therefore $p=0$ on $L_{\omega}$, contrary to $p(\omega)>0$.

\section{Supporting Pareto optimal allocations}

For any $m$-tuple of continuous linear functionals $f=\left(f_{1}, \ldots, f_{m}\right)$ on $L$, recall that, in view of the assumption $\mathbf{A} \mathbf{2}$ and the continuity of the $f_{i}$, the Riesz-Kantorovich functional $\mathcal{R}_{f}$ is finite-valued and for each $z \in L_{+}$is defined by the formula

$$
\mathcal{R}_{f}(z)=\sup \left\{\sum_{i=1}^{m} f_{i}\left(z_{i}\right): z_{i} \geq 0 \text { for each } i \text { and } \sum_{i=1}^{m} z_{i}=z\right\} .
$$

The main result of this section is the following:

Theorem 4.1. In our model the following conditions are equivalent:

(1) For each $\omega$-proper economy with total endowment $\omega>0$, every weakly Pareto optimal allocation is a nontrivial quasi-valuation equilibrium for some $\tau$-continuous price.

(2) For any list of continuous linear functionals $f=\left(f_{1}, \ldots, f_{m}\right)$ such that $f_{i}(\omega)>0$ for each $i$ and $\mathcal{R}_{f}$ is exact at $\omega$ with respect to some $x=\left(x_{1}, \ldots, x_{m}\right) \in \mathcal{A}_{\omega}^{m}$, there exists some $\left(\lambda_{1}, \ldots, \lambda_{m}\right) \gg 0$ such that the Riesz-Kantorovich formula of the $m$-tuple of continuous linear functionals $\left(\lambda_{1} f_{1}, \ldots, \lambda_{m} f_{m}\right)$ is exact at $\omega$ with respect to $x$ and pointwise $\omega$-proper at $\omega$.

The proof will be done in three separate lemmas.

Lemma 4.2. In Theorem 4.1: (1) $\Longrightarrow(2)$.

Proof. Pick $m$ continuous linear functionals $f=\left(f_{1}, \ldots, f_{m}\right)$ such that $f_{i}(\omega)>0$ holds for each $i=$ $1, \ldots, m$ and let $x=\left(x_{1}, \ldots, x_{m}\right) \in\left(L_{+}\right)^{m}$ be such that $\omega=\sum_{i=1}^{m} x_{i}$ and $\mathcal{R}_{f}(\omega)=\sum_{i=1}^{m} f_{i}\left(x_{i}\right)$. We have clearly defined an $\omega$-proper economy with total endowment $\omega$ and linear utility functions $f_{1}, \ldots, f_{m}$, for which $x$ is a Pareto optimal allocation. So, according to our hypothesis, there exists some $f^{*} \in L^{\prime}$ that supports the Pareto optimal allocation $x$ as a nontrivial quasi-valuation equilibrium. In other words, $f^{*}(\omega)>0$ and for each $i$ the bundle $x_{i}$ minimizes $f^{*}\left(y_{i}\right)$ under the

\footnotetext{
${ }^{7}$ That is, the order interval $[-\omega, \omega]$ is radial at the origin.
} 
constraint $\widehat{f}_{i}\left(y_{i}\right) \geq \widehat{f}_{i}\left(x_{i}\right)$. Notice that $f_{i}(\omega)>0$ implies $\widehat{f}_{i}\left(x_{i}+\omega\right)>\widehat{f}_{i}\left(x_{i}\right)$. So, for each $i$ there exists $\lambda_{i} \geq 0$ such that $f^{*} \in \lambda_{i} \partial \widehat{f_{i}}\left(x_{i}\right)=\partial\left(\lambda_{i} \widehat{f}_{i}\right)\left(x_{i}\right)$. Moreover, since $f^{*}\left(y_{i}\right) \geq f^{*}\left(x_{i}\right)$ for every $y_{i} \in L_{+}$implies $f^{*}=0$, one deduces that $\lambda_{i}>0$ for each $i$.

Let $g=\left(\lambda_{1} f_{1}, \ldots, \lambda_{m} f_{m}\right)$. We first claim that $\mathcal{R}_{g}(\omega)=\sum_{i=1}^{m} \lambda_{i} f_{i}\left(x_{i}\right)$. To see this, let $\sum_{i=1}^{m} y_{i}=\omega$ with $y_{i} \geq 0$ for each $i$. Using $f^{*} \in \partial\left(\lambda_{i} \widehat{f}_{i}\right)\left(x_{i}\right)$, we get $f^{*}\left(y_{i}-x_{i}\right) \geq \lambda_{i} f_{i}\left(y_{i}\right)-\lambda_{i} f_{i}\left(x_{i}\right)$. This implies

$$
\sum_{i=1}^{m} \lambda_{i} f_{i}\left(y_{i}\right) \leq \sum_{i=1}^{m} \lambda_{i} f_{i}\left(x_{i}\right)+\sum_{i=1}^{m} f^{*}\left(y_{i}-x_{i}\right)=\sum_{i=1}^{m} \lambda_{i} f_{i}\left(x_{i}\right) .
$$

Therefore, $\mathcal{R}_{g}$ is exact at $\omega$ with respect to $x=\left(x_{1}, \ldots, x_{m}\right)$.

From Theorem 2.3, we see that $f^{*} \in \partial\left(\nabla_{i=1}^{m} \lambda_{i} \widehat{f}_{i}\right)(\omega)$. This implies that for every $\omega^{\prime} \in L_{+}$we have

$$
f^{*}\left(\omega^{\prime}\right)-f^{*}(\omega) \geq \mathcal{R}_{g}\left(\omega^{\prime}\right)-\mathcal{R}_{g}(\omega) .
$$

Consequently, as $0=f^{*}(\omega-\omega)<f^{*}(\omega)$, letting $\Gamma_{\omega}=\left\{\omega^{\prime} \in L: f^{*}\left(\omega^{\prime}\right)<f^{*}(\omega)\right\}$, we see that $\Gamma_{\omega}$ satisfies the required properties for $\mathcal{R}_{g}$ to be $\omega$-pointwise proper at $\omega$.

Before proving the reverse implication, let us recall a convexity result due to Podczeck [35]. In order to keep this paper self-contained, we state the result and present a simplified proof.

Lemma 4.3 (Podczeck). Let $(L, \tau)$ be an ordered topological vector space, let $M$ be a vector subspace of $L$ (endowed with the induced order), let $Y$ be an open and convex subset of $L$ such that $Y \cap M_{+} \neq \varnothing$ and let $y \in \bar{Y} \cap M_{+}$. If $p$ is a linear functional on $M$ satisfying

$$
p(y) \leq p(z) \text { for all } z \in Y \cap M_{+},
$$

then there exists some $\pi \in L^{\prime}$ such that $\left.\pi\right|_{M} \leq p$ and

$$
p(y)=\pi(y) \leq \pi(z) \text { for all } z \in Y .
$$

Proof. Consider the set

$$
Z=\left\{\left(y^{\prime}, r\right): \exists z \in Y \text { and } m \in M_{+} \text {such that } y^{\prime}=z-m \text { and } r>p(m)\right\} .
$$

From our assumptions, it follows that $Z$ is a non-empty, open, and convex subset of $L \times \mathbb{R}$ and that $(0, p(y)) \notin Z$. By the classical separation theorem, there exists a non-zero $(\pi, t) \in L^{\prime} \times \mathbb{R}$, such that

$$
t p(y)<\pi(z)-\pi(m)+t r,
$$

for all $z \in Y$, for all $m \in M_{+}$, and for all $r>p(m)$.

We claim that $t>0$. To see this, assume first that $t<0$. Then letting $m=y$ and $r=p(m)+1$ in $(\star)$ we get $t p(y) \leq \pi(z)-\pi(y)+t[p(y)+1]$ or $-t \leq \pi(z)-\pi(y)$ for all $z \in Y$. Since $y \in \bar{Y}$, we get $-t \leq 0$ or $t \geq 0$, a contradiction. Now assume that $t=0$. Then $(\star)$ implies that $0<\pi(z)-\pi(m)$ for all $z \in Y$ and all $m \in M_{+}$. Since $Y \cap M_{+} \neq \varnothing$, we see that $0<0$, which is impossible.

Replacing $\pi$ by $\frac{\pi}{t}$, we can assume that $t=1$. This and $(\star)$ imply

$$
p(y) \leq \pi(z)-\pi(m)+p(m),
$$

for all $z \in Y$ and all $m \in M_{+}$. Since $y \in \bar{Y}$, we get

$$
[p-\pi](m) \geq[p-\pi](y),
$$

for all $m \in M_{+}$. Since $M_{+}$is a cone, it follows that $[p-\pi](m) \geq 0$ for all $m \in M_{+}$, i.e., $p \geq\left.\pi\right|_{M} \geq 0$. Letting $m=y \in M_{+}$in $[p-\pi](m) \geq 0$, we get $\pi(y) \leq p(y)$. Also, letting $m=0$ in ( $\left.\star \star \star\right)$, we get $p(y) \leq \pi(y)$. Therefore, $\pi(y)=p(y)$, and the proof is finished. 
We are now ready to finish the proof of Theorem 4.1 .

Lemma 4.4. In Theorem 4.1: (2) $\Longrightarrow(1)$.

Proof. Assume that $x=\left(x_{1}, \ldots, x_{m}\right)$ is a weakly Pareto optimal allocation for an $\omega$-proper economy with $m$ consumers and total endowment $\omega>0$. Recall that the ordered subspace

$$
L_{\omega}=\bigcup_{\lambda>0} \lambda[-\omega, \omega],
$$

endowed with the finest locally convex topology on $L_{\omega}$ for which every order interval is topologically bounded is normable. More precisely, the gauge $p_{\omega}$ of $[-\omega, \omega]$ is a norm on $L_{\omega}$ and $\omega$ is an interior point of $L_{\omega}^{+}$. As the order intervals of $L$ are $\tau$-bounded, observe incidentally that the order topology is finer than the topology induced by $\tau$ on $L_{\omega}$. We will denote by $L_{\omega}^{\prime}$ the topological dual of $L_{\omega}$ for the order topology.

Let us now consider $\mathcal{E}_{\left.\right|_{\omega}}$ the economy deduced from $\mathcal{E}$ where consumers are restricted to $L_{\omega}$. Clearly, $x=\left(x_{1}, \ldots, x_{m}\right)$ is a weakly Pareto optimal allocation of $\mathcal{E}_{\left.\right|_{\omega}}$. Since the order topology on $L_{\omega}$ is finer than $\tau$ when restricted to $L_{\omega}$, it follows that the economy $\mathcal{E}_{\left.\right|_{\omega}}$ is $\omega$-proper. In addition, we claim that each restricted preferred set on $L_{\omega}$ at $x_{i}$ has a nonempty interior. To see this, choose a $\tau$-neighborhood $V$ of zero such that $x_{i}+\omega+V \subset \widehat{P}_{i}\left(x_{i}\right)$. Since $V$ is also a $\|\cdot\|_{\omega}$-neighborhood of zero, there exists some $0<\epsilon<1$ such that $B(0, \epsilon) \subset V$, and so $x_{i}+\omega+B(0, \epsilon) \subset \widehat{P}_{i}\left(x_{i}\right)$. Since $[-\omega, \omega]$ is a $\|\cdot\|$-neighborhood of zero, it follows that $x_{i}+\omega+B(0, \epsilon) \subset x_{i}+[0,2 \omega] \subset L_{\omega}^{+}$, and thus $x_{i}+\omega+B(0, \epsilon) \subset \widehat{P}_{i}\left(x_{i}\right) \cap L_{\omega}^{+}=\left.P_{i}\right|_{L_{\omega}}\left(x_{i}\right)$.

The local nonsatiation of preferences (in $\mathcal{E}_{\left.\right|_{\omega}}$ ) at each $x_{i}$ follows from the $\omega$-properness of the preferences. Indeed, observe that for each $0<\lambda<1$ we have $\lambda x_{i}+(1-\lambda) \omega \in P_{i}\left(x_{i}\right) \cap L_{\omega}=$ $\left.P_{i}\right|_{L_{\omega}}\left(x_{i}\right)$.

Now, as in Debreu [18], there exists some non-zero $p \in L_{\omega}^{\prime}$ such that $(x, p)$ is a quasi-valuation equilibrium of $\mathcal{E}_{\left.\right|_{L_{\omega}}}$. That is,

$$
p(z) \geq p\left(x_{i}\right) \text { for all } z \in P_{i}\left(x_{i}\right) \cap L_{\omega} .
$$

Looking at the proof of Proposition 3.1, one sees immediately that $(x, p)$ is a nontrivial quasivaluation equilibrium and that $p(\omega)>0$.

Applying Lemma 4.3 with $M=L_{\omega}, Y$ the interior of the convex set $\widehat{P}_{i}\left(x_{i}\right), y=x_{i}$, and $p$ the price satisfying $(\dagger)$, we obtain for each $i$ a $\tau$-continuous linear functional $\pi_{i}$ on $L$ such that $\pi_{\left.i\right|_{L_{\omega}}} \leq p$ and

$$
p\left(x_{i}\right)=\pi_{i}\left(x_{i}\right) \leq \pi_{i}\left(y_{i}\right) \text { for all } y_{i} \in \widehat{P}_{i}\left(x_{i}\right) .
$$

Since $x_{i}+\omega \in \operatorname{int} \widehat{P}_{i}\left(x_{i}\right)$, one deduces that $\pi_{i}(\omega)>0$. On the other hand, $y_{i} \in L_{+}$for each $i$ and $\sum_{i \in I} y_{i}=\omega$ imply

$$
\sum_{i=1}^{m} \pi_{i}\left(y_{i}\right) \leq p\left(\sum_{i=1}^{m} y_{i}\right)=p(\omega)=p\left(\sum_{i=1}^{m} x_{i}\right)=\sum_{i=1}^{m} \pi_{i}\left(x_{i}\right) .
$$

From this it follows that for the list $\pi=\left(\pi_{1}, \ldots, \pi_{m}\right) \in\left(L^{\prime}\right)^{m}$ the Riesz-Kantorovich formula $\mathcal{R}_{\pi}$ is exact at $\omega$ with respect to $x=\left(x_{1}, \ldots, x_{m}\right)$. In view of our assumption (2), there exist $\left(\lambda_{1}, \ldots, \lambda_{m}\right) \gg 0$ such that if $g=\left(\lambda_{1} \pi_{1}, \ldots, \lambda_{m} \pi_{m}\right)$, then $\mathcal{R}_{g}(\omega)=\sum_{i=1}^{m} \lambda_{i} \pi_{i}\left(x_{i}\right)$ and $\mathcal{R}_{g}$ is $\omega$-pointwise proper at $\omega$.

Notice that $\mathcal{R}_{g}(\omega)>0$, hence $\mathcal{R}_{g}(2 \omega)=2 \mathcal{R}_{g}(\omega)>\mathcal{R}_{g}(\omega)$. The concavity of $\mathcal{R}_{g}$ implies that local no-satiation at every $\omega^{\prime} \in L_{+}$with $\mathcal{R}_{g}\left(\omega^{\prime}\right)=\mathcal{R}_{g}(\omega)$. 
Let $\bar{p} \in L^{\prime}$ be such that $\bar{p}(\omega)>0$ and

$$
\mathcal{R}_{g}\left(\omega^{\prime}\right)>\mathcal{R}_{g}(\omega) \Longrightarrow \bar{p}\left(\omega^{\prime}\right) \geq \bar{p}(\omega) .
$$

In view of the previous remark, we have

$$
\mathcal{R}_{g}\left(\omega^{\prime}\right) \geq \mathcal{R}_{g}(\omega) \Longrightarrow \bar{p}\left(\omega^{\prime}\right) \geq \bar{p}(\omega) .
$$

Now observe that if $\omega^{\prime}=y+\sum_{j \neq i} x_{j}$, then $y \in P_{i}\left(x_{i}\right)$ implies $\mathcal{R}_{g}\left(\omega^{\prime}\right) \geq \mathcal{R}_{g}(\omega)$. Hence $y \in P_{i}\left(x_{i}\right)$ implies $\bar{p}(y) \geq \bar{p}\left(x_{i}\right)$, which proves that $(x, \bar{p})$ is a quasi-valuation equilibrium of the $\omega$-proper economy. This quasi-valuation equilibrium is nontrivial in view of Proposition 3.1.

\section{Decentralizing Edgeworth equilibrium allocations}

The result we will prove in this section is the following:

Theorem 5.1. Consider the following three statements.

(1) If $\left(f_{1}, \ldots, f_{m}\right)$ is a list of continuous linear functionals such that $f_{i}(\omega)>0$ for each $i$ and $\mathcal{R}_{f}$ is exact at $\omega$ with respect to some $x=\left(x_{1}, \ldots, x_{m}\right) \in \mathcal{A}_{\omega}^{m}$, then the Riesz-Kantorovich functional $\mathcal{R}_{f}$ is $\omega$-proper at $\omega$.

(2) If $\mathcal{E}$ is a proper economy on $L$ with total endowment $\omega>0$, then for any Edgeworth equilibrium $x$ of $\mathcal{E}$ there exists some $p \in L^{\prime}$ such that $(x, p)$ is a nontrivial quasi-equilibrium.

(3) If $\left(f_{1}, \ldots, f_{m}\right)$ is a list of continuous linear functionals such that $f_{i}(\omega)>0$ for each $i$, and $\mathcal{R}_{f}$ is exact at $\omega$ with respect to some $x=\left(x_{1}, \ldots, x_{m}\right) \in \mathcal{A}_{\omega}^{m}$, then the Riesz-Kantorovich functional $\mathcal{R}_{f}$ is pointwise $\omega$-proper at $\omega$.

Then the following implications hold true: (1) $\Longrightarrow(2) \Longrightarrow(3)$.

In the next proposition, we give sufficient conditions for decentralizing any Edgeworth allocation of a proper economy as a personalized quasi-equilibrium ${ }^{8}$ with respect to a list of continuous personalized prices $\pi=\left(\pi_{1}, \ldots, \pi_{m}\right)$ such that $\pi_{i}(\omega)>0$ for each $i=1, \ldots, m$. More precisely, the following proposition extends statement (1) of Theorem 7.5 in Aliprantis-Tourky-Yannelis [11].

Proposition 5.1. Let $\mathcal{E}=\left(X_{i}, P_{i}, \omega_{i}\right)_{i \in I}$ be a proper economy with total endowment $\omega>0$. If $\left(x_{1}, \ldots, x_{m}\right)$ is an Edgeworth equilibrium, then there exists a list $\left(\pi_{1}, \ldots, \pi_{m}\right)$ of $\tau$-continuous linear functionals satisfying $\pi_{i}(\omega)>0$ for each $i$ and such that:

(1) $\mathcal{R}_{\pi}(\omega)>0$,

(2) $y_{i} \in P_{i}\left(x_{i}\right) \Longrightarrow \mathcal{R}_{\pi}\left(y_{i}\right) \geq \mathcal{R}_{\pi}\left(x_{i}\right)$,

(3) $\mathcal{R}_{\pi}\left(\sum_{i=1}^{m} \alpha_{i} \omega_{i}\right) \leq \sum_{i=1}^{m} \alpha_{i} \mathcal{R}_{\pi}\left(x_{i}\right)$ for all $\alpha \in \mathbb{R}_{+}^{m}$, and

(4) $\mathcal{R}_{\pi}(\omega)=\sum_{i=1}^{m} \mathcal{R}_{\pi}\left(x_{i}\right)=\sum_{i=1}^{m} \pi_{i}\left(x_{i}\right)$.

Proof. Let $\mathcal{E}=\left(X_{i}, P_{i}, \omega_{i}\right)_{i \in I}$ be a proper economy with total endowment $\omega$ and let $\left(x_{1}, \ldots, x_{m}\right)$ be an Edgeworth equilibrium of $\mathcal{E}$. As in the proof of Lemma 4.4, we define the ordered subspace

$$
L_{\omega}=\bigcup_{\lambda>0} \lambda[-\omega, \omega]
$$

and consider the economy $\mathcal{E}_{\left.\right|_{L_{\omega}}}$ obtained from $\mathcal{E}$ when consumers are restricted to $L_{\omega}$. It is easily checked that $x$ is an Edgeworth equilibrium of $\mathcal{E}_{\left.\right|_{L_{\omega}}}$. As already noticed in the proof of Lemma 4.4 , the economy $\mathcal{E}_{\left.\right|_{L_{\omega}}}$ is $\omega$-proper and each restricted preferred set at $x_{i}$ has a nonempty interior for the order topology of $L_{\omega}$. Local nonsatiation of preferences (in $\mathcal{E}_{\left.\right|_{L_{\omega}}}$ ) at each $x_{i}$ follows from

\footnotetext{
${ }^{8}$ As defined in [11].
} 
the $\omega$-properness of preferences. It is then standard (see, for example, [20, Proposition 4.1]) that there is some non-zero $p \in L_{\omega}^{\prime}$ such that $(x, p)$ is a quasi-equilibrium of $\mathcal{E}_{\left.\right|_{L_{\omega}}}$. Looking at the proof of Proposition 3.1, one sees also that $(x, p)$ is a nontrivial quasi-equilibrium and that $p(\omega)>0$. Thus for every $i$ we have

$$
p\left(y_{i}\right) \geq p\left(x_{i}\right)=p\left(\omega_{i}\right) \text { for all } y_{i} \in P_{i}\left(x_{i}\right) \cap L_{\omega} .
$$

As in the proof of Lemma 4.4, applying Lemma 4.3, we get for each $i$ a $\tau$-continuous linear functional $\pi_{i}$ on $L$ such that $\pi_{\left.i\right|_{L_{\omega}}} \leq p$ and

$$
p\left(\omega_{i}\right)=p\left(x_{i}\right)=\pi_{i}\left(x_{i}\right) \leq \pi_{i}\left(y_{i}\right) \text { for all } y_{i} \in \widehat{P}_{i}\left(x_{i}\right),
$$

from which we deduce $\pi_{i}(\omega)>0$. Therefore, for the list $\left(\pi_{1}, \ldots, \pi_{m}\right)$ we have $\mathcal{R}_{\pi}(\omega)>0$, and this establishes (1).

In view of $\pi_{\left.i\right|_{\omega}} \leq p$, we see that $\mathcal{R}_{\pi}\left(x_{i}\right) \leq p\left(x_{i}\right)$, and (2) follows at once from (5.1). On the other hand, for each $\alpha \in \mathbb{R}_{+}^{I}$, we have

$$
\sum_{i=1}^{m} \alpha_{i} \mathcal{R}_{\pi}\left(x_{i}\right) \geq \sum_{i=1}^{m} \alpha_{i} \pi_{i}\left(x_{i}\right)=\sum_{i=1}^{m} \alpha_{i} p\left(x_{i}\right)=p\left(\sum_{i=1}^{m} \alpha_{i} \omega_{i}\right) \geq \mathcal{R}_{\pi}\left(\sum_{i=1}^{m} \alpha_{i} \omega_{i}\right),
$$

which proves (3).

Now, (3) and the super-additivity of $\mathcal{R}_{\pi}$ imply that $\mathcal{R}_{\pi}(\omega)=\sum_{i=1}^{m} \mathcal{R}_{\pi}\left(x_{i}\right)$. One then deduces from (5.1) that

$$
p(\omega)=\sum_{i=1}^{m} \pi_{i}\left(x_{i}\right) \leq \mathcal{R}_{\pi}(\omega)=\sum_{i=1}^{m} \mathcal{R}_{\pi}\left(x_{i}\right) \leq \sum_{i=1}^{m} p\left(x_{i}\right)=p(\omega),
$$

i.e., $\mathcal{R}_{\pi}(\omega)=\sum_{i=1}^{m} \pi_{i}\left(x_{i}\right)$, which completes the proof of (4) and the proof of the Proposition.

The next lemma states that (1) implies (2) in Theorem 5.1.

Lemma 5.2. Assume that for any finite list of continuous linear functionals $\left(f_{1}, \ldots, f_{m}\right)$ the Riesz-Kantorovich functional $\mathcal{R}_{f}$ is $\omega$-proper at $\omega$ whenever $f_{i}(\omega)>0$ for each $i$ and $\mathcal{R}_{f}$ is exact at $\omega$ with respect to some $x=\left(x_{1}, \ldots, x_{m}\right) \in \mathcal{A}_{\omega}^{m}$.

Then for every Edgeworth equilibrium $\bar{x}$ of any $\omega$-proper economy $\mathcal{E}$ with total endowment $\omega$, there exists $\bar{\pi} \in L^{\prime}$ such that $(\bar{x}, \bar{\pi})$ is a non-trivial quasi-equilibrium.

Proof. Let $\bar{x}$ be an Edgeworth equilibrium of the economy $\mathcal{E}$. Recall that according to the proof of Proposition 5.1 there exist:

(1) some price $p \in L_{\omega}^{\prime}$ such that $(\bar{x}, p)$ is a non-trivial quasi-equilibrium price for the economy $\mathcal{E}_{\left.\right|_{L_{\omega}}}$, and

(2) a list of $\tau$-continuous prices $\pi=\left(\pi_{1}, \ldots, \pi_{m}\right)$ that satisfies the properties listed in Proposition 5.1 and $\pi_{\left.i\right|_{L_{\omega}}} \leq p$ for each $i$.

It follows that $p(\omega)>0$. So, replacing $p$ by $\frac{p}{p(\omega)}$, we can assume that $p(\omega)=1$. Also, according to Proposition 5.1, we have $\mathcal{R}_{\pi}(\omega)>0$, and so by normalizing appropriately we can assume that $\mathcal{R}_{\pi}(\omega)=1$. Now let $X=\left\{\omega^{\prime} \in L_{\omega}^{+}: p\left(\omega^{\prime}\right) \leq p(\omega)\right\}$.

Put $P(\omega)=\left\{\omega^{\prime} \in L_{+}: \mathcal{R}_{\pi}\left(\omega^{\prime}\right)>\mathcal{R}_{\pi}(\omega)=1\right\}$. By the $\omega$-properness of $\mathcal{R}_{\pi}$ at $\omega$, we get the existence of $\widehat{P}(\omega)$ such that $2 \omega$ is a $\tau$-interior point of $\widehat{P}(\omega)$ and $P(\omega)=\widehat{P}(\omega) \cap L_{+}$. Clearly, $\omega \in \widehat{\widehat{P}(\omega)}$. Moreover, if $\omega^{\prime} \in \widehat{P}(\omega) \cap L_{\omega}^{+}$, then $\mathcal{R}_{\pi}\left(\omega^{\prime}\right)>\mathcal{R}_{\pi}(\omega)=p(\omega)=1$. In view of $\pi_{\left.i\right|_{L_{\omega}} \leq p}$ for each $i$, it follows that $p\left(\omega^{\prime}\right)>p(\omega)$. The last observation can be rephrased as $X \cap \widehat{P}(\omega)=\varnothing$. 
Let a non-zero $\bar{\pi} \in L^{\prime}$ separate $\widehat{P}(\omega)$ and $X$. From $\bar{\pi}(2 \omega)>\bar{\pi}(\omega)$, we deduce $\bar{\pi}(\omega)>0$, so that we can assume $\bar{\pi}(\omega)=p(\omega)=1$.

We next claim that $\bar{\pi}_{\left.\right|_{L_{\omega}}}=p$. Indeed, for every $\omega^{\prime} \in L_{\omega}^{+}$we know that:

$$
\text { If } \omega^{\prime} \in L_{\omega} \text { satisfies } p\left(\omega^{\prime}\right) \leq p(\omega) \text {, then } \bar{\pi}\left(\omega^{\prime}\right) \leq \bar{\pi}(\omega) \text {. }
$$

So, there exist two real numbers $\lambda_{1} \geq 0$ and $\lambda_{2} \geq 0$ such that $\lambda_{1}\left[\bar{\pi}\left(\omega^{\prime}\right)-\bar{\pi}(\omega)\right] \leq \lambda_{2}\left[p\left(\omega^{\prime}\right)-p(\omega)\right]$ for every $\omega^{\prime} \in L_{\omega}^{+}$. Recalling that for the order topology of $L_{\omega}$ the bundle $\omega$ is an interior point of $L_{\omega}^{+}$, we deduce that $\left(\lambda_{1}, \lambda_{2}\right) \gg 0$. Letting $\lambda=\lambda_{2} / \lambda_{1}$, we obtain that $\bar{\pi}\left(\omega^{\prime}\right)-\lambda p\left(\omega^{\prime}\right) \leq \bar{\pi}(\omega)-\lambda p(\omega)$ for each $\omega^{\prime} \in L_{\omega}^{+}$. Since $\omega$ is an interior point of $L_{\omega}^{+}$, it follows that $\bar{\pi}=\lambda p$ on $L_{\omega}$. Taking into account that $\bar{\pi}(\omega)=p(\omega)=1$, we conclude that $\lambda=1$, and so $p=\bar{\pi}$ on $L_{\omega}$.

Finally, notice that

$$
x_{i} \in P_{i}\left(\bar{x}_{i}\right) \Longrightarrow \mathcal{R}_{\pi}\left(x_{i}\right) \geq \mathcal{R}_{\pi}\left(\bar{x}_{i}\right) \Longrightarrow \bar{\pi}\left(x_{i}\right) \geq \bar{\pi}\left(\bar{x}_{i}\right)=p\left(\bar{x}_{i}\right)=p\left(\omega_{i}\right)=\bar{\pi}\left(\omega_{i}\right),
$$

which shows that $(\bar{x}, \bar{\pi})$ is a quasi-equilibrium of $\mathcal{E}$. As $\bar{\pi}(\omega)>0$, it follows from Proposition 3.1 that this quasi-equilibrium is nontrivial.

We are now ready to prove that (2) implies (3) in Theorem 5.1.

Lemma 5.3. Assume that for each proper economy $\mathcal{E}$ on $L$ with total endowment $\omega$ and each Edgeworth equilibrium allocation $x$ of $\mathcal{E}$ there exists $p \in L^{\prime}$ such that $(x, p)$ is a nontrivial quasiequilibrium.

Then for any finite list of continuous linear functionals $\left(f_{1}, \ldots, f_{m}\right)$ such that $f_{i}(\omega)>0$ for each $i$ and $\mathcal{R}_{f}$ is exact at $\omega$ with respect to some $x=\left(x_{1}, \ldots, x_{m}\right) \in \mathcal{A}_{\omega}^{m}$, the Riesz-Kantorovich functional $\mathcal{R}_{f}$ is pointwise $\omega$-proper at $\omega$.

Proof. Pick $m$ continuous linear functionals $\left(f_{1}, \ldots, f_{m}\right)$ such that $f_{i}(\omega)>0$ for each $i$ and let $x=\left(x_{1}, \ldots, x_{m}\right) \in L_{+}^{m}$ be such that $\sum_{i=1}^{m} x_{i}=\omega$ and $\mathcal{R}_{f}(\omega)=\sum_{i=1}^{m} f_{i}\left(x_{i}\right)$.

Let us first remark that it follows from the super-additivity of $\mathcal{R}_{f}$ that

$$
\mathcal{R}_{f}(\omega) \geq \sum_{i=1}^{m} \mathcal{R}_{f}\left(x_{i}\right) \geq \sum_{i=1}^{m} f_{i}\left(x_{i}\right)=\mathcal{R}_{f}(\omega) .
$$

This implies that $\mathcal{R}_{f}(\omega)=\sum_{i=1}^{m} \mathcal{R}_{f}\left(x_{i}\right)=\sum_{i=1}^{m} f_{i}\left(x_{i}\right)$ and thus $\mathcal{R}_{f}\left(x_{i}\right)=f_{i}\left(x_{i}\right)$ holds for every $i$. Without loss of generality, we can assume $\mathcal{R}_{f}(\omega)=1$, so that the economy $\mathcal{E}$ with individual endowments $\mathcal{R}_{f}\left(x_{1}\right) \omega, \ldots, \mathcal{R}_{f}\left(x_{m}\right) \omega$ and linear utility functions $f_{1}, \ldots, f_{m}$ is clearly an $\omega$-proper economy with total endowment $\omega$.

We first claim that $x$ is an Edgeworth equilibrium of $\mathcal{E}$. To see this, assume by way of contradiction that there exist $\tau=\left(\tau_{i}\right)_{i=1}^{m} \in[0,1]^{m} \backslash\{0\}$ and $y \in L_{+}^{m}$ such that

$$
\sum_{i=1}^{m} \tau_{i} y_{i}=\sum_{i=1}^{m} \tau_{i} \mathcal{R}_{f}\left(x_{i}\right) \omega=\left[\sum_{i=1}^{m} \tau_{i} f_{i}\left(x_{i}\right)\right] \omega
$$

and $f_{i}\left(y_{i}\right)>f_{i}\left(x_{i}\right)$ for all $\tau_{i}>0$. Clearly,

$$
\sum_{i=1}^{m} \frac{\tau_{i}}{\sum_{i=1}^{m} \tau_{i} f_{i}\left(x_{i}\right)} y_{i}=\omega
$$

and it follows from the super-additivity and the positive homogeneity of $\mathcal{R}_{f}$ that 


$$
\begin{aligned}
1=\mathcal{R}_{f}(\omega) & =\mathcal{R}_{f}\left(\frac{\sum_{i=1}^{m} \tau_{i} y_{i}}{\sum_{i=1}^{m} \tau_{i} f_{i}\left(x_{i}\right)}\right) \\
& \geq \frac{\sum_{i=1}^{m} \tau_{i} \mathcal{R}_{f}\left(y_{i}\right)}{\sum_{i=1}^{m} \tau_{i} f_{i}\left(x_{i}\right)} \\
& \geq \frac{\sum_{i=1}^{m} \tau_{i} f_{i}\left(y_{i}\right)}{\sum_{i=1}^{m} \tau_{i} f_{i}\left(x_{i}\right)} \\
& >\frac{\sum_{i=1}^{m} \tau_{i} f_{i}\left(x_{i}\right)}{\sum_{i=1}^{m} \tau_{i} f_{i}\left(x_{i}\right)}=1
\end{aligned}
$$

a contradiction. This establishes the validity of the claim.

Let now $\pi \in L^{\prime}$ be such that $\pi(\omega)>0$ and for every $y_{i} \in L_{+}$satisfying $f_{i}\left(y_{i}\right)>f_{i}\left(x_{i}\right)$ we have $\pi\left(y_{i}\right) \geq \pi\left(x_{i}\right)=\pi\left(f_{i}\left(x_{i}\right) \omega\right)$. Without loss of generality, we can assume $\pi(\omega)=1$, so that $f_{i}\left(x_{i}\right)=\pi\left(x_{i}\right)>0$ for some $i$. Now for each $i$ let $\lambda_{i}>0$ be such that

$$
\lambda_{i}\left[\pi\left(y_{i}\right)-\pi\left(x_{i}\right)\right] \geq f_{i}\left(y_{i}\right)-f_{i}\left(x_{i}\right) \text { for all } y_{i} \in L_{+} .
$$

Letting $y_{i}$ equal 0 and $2 x_{i}$, we get $\lambda_{i}=1$, so that for each $i$ we have $\pi \in \partial \widehat{f}_{i}\left(x_{i}\right)$. In view of Theorem 2.3, we get $\pi \in \partial\left(\nabla_{i=1}^{m} \widehat{f}_{i}\right)(\omega)$, which implies for every $\omega^{\prime} \in L_{+}$that

$$
\pi\left(\omega^{\prime}\right)-\pi(\omega) \geq \mathcal{R}_{f}\left(\omega^{\prime}\right)-\mathcal{R}_{f}(\omega) .
$$

Since $0=\pi(\omega-\omega)<\pi(\omega)$, letting $\Gamma_{\omega}=\left\{\omega^{\prime} \in L: \pi\left(\omega^{\prime}\right)<\pi(\omega)\right\}$, we see that $\Gamma_{\omega}$ satisfies the required properties for $\mathcal{R}_{f}$ to be $\omega$-pointwise proper at $\omega$.

\section{EXAMPLES}

Most of the currently used commodity spaces are Banach lattices. It is the case for example of $\mathbb{R}^{\ell}$, $C^{0}(\mathbb{R}), L_{p}(\Omega, \Sigma, \mu), 1 \leq p \leq \infty, \mathcal{M}(X)$, the space of countably additive signed measures on a compact metric space $X$, with their natural orders and their norm-topologies. The same space $\mathcal{M}(X)$, endowed with the weak-star topology relative to its predual $C(X),(\mathcal{M}(X), \sigma(\mathcal{M}(X), C(X)))$, is not a topological vector lattice but satisfies Assumptions B1 and B2. The same is true for the space $L$ of right continuous real valued functions of bounded variation defined on $[0,1]$, equipped with a topology such that its topological dual $L^{\prime}$ is a lattice in its order dual and order intervals are weakly compact. Hindy, Huang and Kreps [26] exhibit a family of norm topologies on $L$ which satisfy this requirement. It is precisely in order to get an equilibrium existence theorem for these commodity-price dualities, especially appropriate for modeling differentiation of commodities (resp. consumption patterns in a continuous time setting), that were elaborated the previous assumptions. Under such assumptions, it follows from the Riesz-Kantorovich theorem that the Riesz-Kantorovich functional satisfies any desired property referred to in Theorems 4.1 and 5.1. If one notices that this paper deals with non-ordered preferences and does not assume any monotonicity of preferences, the equilibrium existence results which can be obtained using Theorems 4.1 or 5.1 generalize most of the equilibrium existence results which can be found in the literature. 
We now present a few examples of commodity spaces that satisfy Assumptions $\mathbf{A 1}$ and $\mathbf{A 2}$ and are not vector lattices. For models defined on such commodity spaces, Theorems 4.1 and 5.1 give useful criteria in order to know if one can hope to get an equilibrium existence theorem.

Before we present our examples we state a result of T. Andô that characterizes the Decomposition Property.

Theorem 6.1 (Andô [13]). Let $L$ be an ordered Banach space with a closed generating cone whose intervals are norm bounded. Then $L$ has the Riesz Decomposition Property if and only if its norm dual $L^{\prime}$ has likewise the Riesz Decomposition Property.

Example 6.2. Consider the vector space

$$
L=\left\{f \in C[0,2]: \quad f(1)=\frac{1}{2}[f(0)+f(2)]\right\} .
$$

Clearly, $L$ is a closed vector subspace of the Banach lattice $C[0,2]$, where $C[0,2]$ is equipped with the sup norm $\|f\|_{\infty}=\sup _{x \in[0,2]}|f(x)|$. The ordered vector space has the following properties.

(1) $L$ has order units; for instance constant function $\mathbf{1} \in L$ is an order unit.

(2) The positive cone $L_{+}$is closed, generating, with a non-empty interior.

(3) $L$ is not a vector lattice.

(4) $L$ satisfies the Riesz Decomposition Property. ${ }^{9}$

(5) The order intervals are norm bounded.

In particular, it should be clear from the above properties that assumptions $\mathbf{A} 1$ and $\mathbf{A 2}$ are satisfied.

Moreover, in this case the Decomposition Property guarantees that the Riesz-Kantorovich functionals are linear and continuous (since the closed unit ball of $L$ is the order interval $[-\mathbf{1}, \mathbf{1}]$ ). This implies that for any $\omega>0$ and every $m$-tuple $\left(f_{1}, \ldots, f_{m}\right)$ of continuous linear functionals satisfying $f_{i}(\omega)>0$ its Riesz-Kantorovich functional is $\omega$-proper at $\omega$.

Example 6.3. Let $L=C^{k}[0,1]$, the vector space of all real-valued functions on $[0,1]$ which are $k$ times continuously differentiable. With the pointwise ordering and the sup norm, $L$ is an ordered topological vector space such that:

(1) $L$ is not a vector lattice.

(2) $L$ has order units, for instance $\mathbf{1} \in L$ (and hence the positive cone $L_{+}$is generating).

(3) The positive cone is norm closed.

(4) The order intervals are norm bounded.

(5) $L$ has the Riesz decomposition property.

To see that $L$ has the Riesz Decomposition Property, notice that since $L$ is norm dense in $C[0,1]$, it follows that the norm dual of $L$ is Riesz isomorphic to the norm dual of $C[0,1]$. This implies that $L^{\prime}$ is a vector lattice and so, according to Andô's Theorem 6.1, $L$ has the Riesz decomposition property.

Clearly, assumptions $\mathbf{A} \mathbf{1}$ and $\mathbf{A 2}$ are satisfied. Also, as noticed above, $L^{\prime}$ is a vector lattice and hence the Riesz-Kantorovich functionals are all linear. In particular, they are $\omega$-proper at any $\omega>0$.

\footnotetext{
${ }^{9}$ This was shown by I. Namioka [33, p. 45]; see also [34, Example 1.7, p. 14].
} 
In models of finance, $L$ is a portfolio space. As each portfolio is associated with a random payoff, an investor expects to receive when holding the portfolio, it is natural to (re)-order $L$ by the portfolio dominance order, as introduced in Aliprantis-Brown-Polyrakis-Werner [4]. Given an ordered vector payoff space $X$ and a one-to-one linear operator $R: L \rightarrow X$, the vector space $L$ is ordered by the portfolio ordering

$$
z \geq_{R} z^{\prime} \text { whenever } R(z) \geq R\left(z^{\prime}\right) .
$$

If moreover, investors are constrained to have non-negative end-of-period wealth, their portfolio set is equal to the cone of positive payoff portfolios that we will denote by $K$. The three next examples address this interpretation.

Example 6.4. Let $L=\mathbb{R}^{\ell}$ be ordered by a closed convex pointed and generating cone $K$. It follows that $K$ has a non-empty interior and that the order intervals of $L$ are compact. In this case, it is well-known that $K$ has the decomposition property if and only if $K$ has exactly $\ell$ extremal rays, i.e., if and only if $K$ is a lattice cone.

Now fix $\omega>0$ (i.e., $\omega \neq 0$ and $\omega \in K$ ) and pick $m$ linear functionals $\left(f_{1}, \ldots, f_{m}\right)$ such that $f_{i}(\omega)>0$ for each $i$. If $\omega$ is in the interior of $K$, then $\mathcal{R}_{f}$ is $\omega$-proper at $\omega$.

For the points $\omega$ that do not belong to the interior of $K$, we now consider three cone cases.

CASE 1: The cone $K$ is polyhedral ${ }^{10}$ with exactly $\ell$ extremal rays.

In this case $K$ is a lattice cone and the Riesz-Kantorovich functionals are linear and consequently are $\omega$-proper at $\omega$.

CASE 2: The cone $K$ is polyhedral with a number of extremal rays exceeding $\ell$.

In this case we have the following properties.

(1) $K$ is closed.

(2) $\mathcal{R}_{f}$ is "linear" on every $(\ell-1)$-face of the cone.

(3) $\mathcal{R}_{f}$ need not be linear on $K$.

(4) $\mathcal{R}_{f}$ is continuous on $K$. (For this conclusion we need Theorems 10.2 and 20.5 in [36].)

(5) The set $R(\omega):=\left\{\omega^{\prime} \in K: R_{f}\left(\omega^{\prime}\right) \geq R_{f}(\omega)\right\}$ is a polyhedral convex set. (For this conclusion see Theorem 19.3 and Corollary 19.3.4 in [36].)

We claim the following.

- Assume that $\mathbb{R}^{\ell}$ is ordered by a (closed), pointed and generating, polyedral convex cone $K$ that has at least $(\ell+1)$ extremal rays. For $\omega>0$ and any finite list $f=\left(f_{1}, \ldots, f_{m}\right)$ of linear functionals on $L$ such that $f_{i}(\omega)>0$ for each $i$, there exists a convex and open set $\widehat{P}(\omega)$ containing $2 \omega$ such that if $P(\omega)=\left\{\omega^{\prime} \in K: \mathcal{R}_{f}\left(\omega^{\prime}\right)>\mathcal{R}_{f}(\omega)\right\}$ then $P(\omega)=\widehat{P}(\omega) \cap K$. In particular, $\mathcal{R}_{f}$ is $\omega$-proper at $\omega$.

Proof. Express the set $R(\omega)$ as the set of solutions to a certain system of inequalities, say $R(\omega)=$ $\left\{z \in \mathbb{R}^{\ell}: a^{i} \cdot z \geq \alpha^{i}\right.$ for all $\left.i \in I\right\}$, where the finite set $I$ of linear inequalities is minimal. In view of the positive homogeneity of $\mathcal{R}_{f}$, one can assume $\alpha^{i} \geq 0$ for each $i$. Set $I^{\prime}=\left\{i \in I: \alpha^{i}>0\right\}$. Since $\mathcal{R}_{f}(\omega)>0$, it follows that $0 \notin R(\omega)$ and hence $I^{\prime}$ is nonempty. Let us denote by $I(\omega)$ the level set $I(\omega)=\left\{\omega^{\prime} \in K: \mathcal{R}_{f}\left(\omega^{\prime}\right)=\mathcal{R}_{f}(\omega)\right\}$ and define

$$
J(\omega)=\left\{z \in R(\omega): a^{i} \cdot z=\alpha^{i} \text { for some } i \in I^{\prime}\right\} .
$$

\footnotetext{
${ }^{10}$ In $\mathbb{R}^{\ell}$, a polyhedral convex cone is a set which can be expressed as the intersection of finitely many closed half-space whose boundary hyperplanes pass through the origine. Equivalently, if the cone is pointed then it has finitely many extremal rays; see [23, Proposition 3.2.2].
} 
Claim 1. $I(\omega)=J(\omega)$.

Assume first $z \in I(\omega)$ and $z \notin J(\omega)$. So, for each $i \in I^{\prime}$ we have $a^{i} \cdot z>\alpha^{i}$. This implies that there is some $0<\lambda<1$ such that $a^{i} \cdot \lambda z>\alpha^{i}$ for all $i \in I^{\prime}$. Now if $i \notin I^{\prime}$, then $\alpha^{i}=0$ and so $a^{i} \cdot(\lambda z) \geq 0$ and therefore $\lambda z \in R(\omega)$. On the other hand, $z \in I(\omega)$ implies

$$
\mathcal{R}_{f}(\omega)=\mathcal{R}_{f}(z)>\lambda \mathcal{R}_{f}(z)=\mathcal{R}_{f}(\lambda z) \geq \mathcal{R}_{f}(\omega),
$$

which is a contradiction

Conversely, assume $z \in J(\omega)$ and $z \notin I(\omega)$. It follows that $\mathcal{R}_{f}(z)>\mathcal{R}_{f}(\omega)$. So, if $\lambda=\frac{\mathcal{R}_{f}(\omega)}{\mathcal{R}_{f}(z)}$, then $0<\lambda<1$ and $\mathcal{R}_{f}(\lambda z)=\mathcal{R}_{f}(\omega)$. Hence $\lambda z \in I(\omega) \subset R(\omega)$ and thus $a^{i} \cdot \lambda z \geq \alpha^{i}$ for all $i \in I$. Now notice that since $z \in J(\omega)$, there is some $i$ such that $a^{i} \cdot z=\alpha^{i}>0$. In particular for the vector $\lambda z \in R(\omega)$ we have $a^{i} \cdot \lambda z=\lambda a^{i} \cdot z<\alpha^{i}$, contrary to $\lambda z \in R(\omega)$.

Clearly, Claim 1 implies $P(\omega)=R(\omega) \cap\left\{z \in \mathbb{R}^{\ell}: a^{i} \cdot z>\alpha^{i}\right.$ for all $\left.i \in I^{\prime}\right\}$. We now define

$$
\widehat{P}(\omega)=\left\{z \in \mathbb{R}^{\ell}: a^{i} \cdot z>\alpha^{i} \text { for all } i \in I^{\prime}\right\} .
$$

Now note that $\widehat{P}(\omega)$ is a nonempty convex open set and that $P(\omega) \subset \widehat{P}(\omega) \cap K$.

Claim 2. $2 \omega \in \widehat{P}(\omega)$.

To see this, let $I^{\prime \prime}=\left\{i \in I: a^{i} \cdot \omega=0\right\}$. Note that $i \in I^{\prime}$ implies $i \notin I^{\prime \prime}$ (equivalently, $i \in I^{\prime \prime}$ implies $\left.i \notin I^{\prime}\right)$. Moreover, since $\omega \in R(\omega)$, for every $i \in I^{\prime}$ we must have $a^{i} \cdot \omega \geq \alpha^{i}>0$ and consequently $a^{i} \cdot 2 \omega>\alpha^{i}$.

Claim 3. For every $i \notin I^{\prime}$, there is some $u \in R(\omega)$ such that $a^{i} \cdot u=0$. Consequently, for any such $i$, we have $a^{i} \cdot z \geq 0$ for all $z \in K$.

Recall first that if for some $i \in I$ one has $a^{i} \cdot z>\alpha^{i}$ for all $z \in R(\omega)$, then the inequality $a^{i} \cdot z \geq \alpha^{i}$ can be removed from the expression of $R(\omega)$ as $R(\omega)=\left\{z \in \mathbb{R}^{\ell}: a^{i} \cdot z \geq \alpha^{i}\right.$ for all $\left.i \in I\right\}$. Since the finite set of linear inequalities $I$ is minimal, this proves the first assertion.

To prove the second assertion, assume by way of contradiction that there exist $i \notin I^{\prime}$ and $z \in K$ satisfying $a^{i} \cdot z<0$. Let $u \in R(\omega)$ be such that $a^{i} \cdot u=0$. Since $\mathcal{R}_{f}(u) \geq \mathcal{R}_{f}(\omega)>0$, it follows from the continuity at $u$ of $\mathcal{R}_{f}$ that the exists some $z^{\prime} \in K$ such that $a^{i} \cdot z^{\prime}<0$ and $\mathcal{R}_{f}\left(z^{\prime}\right)>0$. Using the positive homogenity of $\mathcal{R}_{f}$, we can find some $\lambda \geq 1$ such that $\mathcal{R}_{f}\left(\lambda z^{\prime}\right) \geq \mathcal{R}_{f}(\omega)$. Hence $a^{i} \cdot\left(\lambda z^{\prime}\right) \geq 0$, which implies $a^{i} \cdot z^{\prime} \geq 0$, contrary to $a^{i} \cdot z^{\prime}<0$.

Claim 4. $\widehat{P}(\omega) \cap K \subset P(\omega)$.

Let $z \in \widehat{P}(\omega) \cap K$. In view of the definition of $\widehat{P}(\omega)$, we have only to prove that $z \in R(\omega)$, i.e., that $z$ satisfies all the inequalities defining $R(\omega)$. For $i \in I^{\prime}$, this follows from $z \in \widehat{P}(\omega)$; for $i \notin I^{\prime}$, this follows from $z \in K$ and the previous claim.

\section{CASE 3: The cone $K$ is not polyhedral.}

In the "ice cream cone" case $K=\left\{\left(x_{1}, x_{2}, x_{3}\right) \in \mathbb{R}^{3}: x_{3} \geq \sqrt{x_{1}^{2}+x_{1}^{2}}\right\}$ on $\mathbb{R}^{3}$, one can construct (as in [8] and [31]) a two-consumer proper linear exchange economy that does not have any non-trivial quasi-equilibrium. It can be shown that in such an economy for certain linear utility functions the Riesz-Kantorovich functional $\mathcal{R}_{f}$ does not satisfy the properties required at $\omega$ in Theorems 4.1 and 5.1 . 
Example 6.5. Let $L=\ell_{p}$, where $1 \leq p \leq \infty$ and consider the ice cream cones

$$
\begin{aligned}
K_{p} & =\left\{\left(x_{1}, x_{2}, \ldots\right) \in \ell_{p}: x_{1} \geq\left[\sum_{k=2}^{\infty}\left|x_{k}\right|^{p}\right]^{\frac{1}{p}}\right\}, 1 \leq p<\infty, \text { and } \\
K_{\infty} & =\left\{\left(x_{1}, x_{2}, \ldots\right) \in \ell_{\infty}: x_{1} \geq\left|x_{k}\right| \text { for all } k \geq 2\right\} .
\end{aligned}
$$

For the Hilbert space case (i.e., $p=2$ ) one can verify the following properties.

(1) The positive cone $K_{2}$ is closed and has a non-empty interior-and hence is generating.

(2) The cone $K_{2}$ is not a lattice cone.

(3) The order intervals are norm bounded.

(4) The dual cone of $K_{2}$ coincides with $K_{2}$.

This is an example of an ordered vector space that satisfies Assumptions A1 and A2. In this case, for each $\omega$ in the interior of $K_{2}$ any Riesz-Kantorovich functional $\mathcal{R}_{f}$ that satisfies $\mathcal{R}_{f}(\omega)>0$ is automatically $\omega$-proper at $\omega$.

Example 6.6. Let $L$ be the vector space $\Phi$ of all eventually zero sequences equipped with the inductive limit topology $\xi$ generated by the family of all its finite dimensional vector subspaces ${ }^{11}$ and ordered by a (convex) cone $K$ on which we make the following hypothesis:

- $K=\bigcup_{n=1}^{\infty} K_{n}$ is pointed (i.e., $K \cap(-K)=\{0\}$ ), generating (i.e., $\left.K-K=\Phi\right)$, and is the union of a countable family of finite dimensional polyhedral convex cones $K_{n}$ such that each $K_{n}$ is an extremal subset ${ }^{12}$ of $K_{n+1}$.

As proved in [7], the cone $K$ is $\xi$-closed and the order-intervals of $\Phi$ are $\xi$-compact. Moreover, for $\omega>0$ (i.e., $\omega \in K$ and $\omega \neq 0)$ and any finite list $f=\left(f_{1}, \ldots, f_{m}\right)$ of continuous linear functionals on $(E, \xi)$ such that $f_{i}(\omega)>0$ for each $i$, the Riesz-Kantorovich functional $\mathcal{R}_{f}$ is $\omega$-proper at $\omega$.

This setting extends the case where, as in Aliprantis-Brown-Polyrakis-Werner [4], $K$ is a Yudin cone. It is used in [7] for proving the existence of a non-trivial quasi-equilibrium of an $\omega$-proper financial economy $\mathcal{E}=\left(\left(\Phi, \xi, \geq_{R}\right),\left(K, P_{i}, \omega_{i}\right)_{i=1}^{m}\right)$ with $\xi$-continuous individual preferences $P_{i}$.

Our last example extends to uncertainty the framework designed by Hindy, Huang and Kreps [26] for modelling consumption patterns in a continuous time setting. It shows how to recover an equilibrium existence result of Bank and Riedel [17] under weaker assumptions on the preferences of the agents.

Example 6.7. Given a filtered probability space $\left(\Omega, \mathcal{F}, \mathcal{F}_{t 0 \leq t \leq 1}, P\right)$, let $L+$ be the set of all adapted stochastic processes with positive, increasing and right continuous sample paths and the positive cone of the vector lattice $L=L_{+}-L_{+}$of all adapted stochastic processes with integrable total variation. When $L$ is equipped with a topology which generalizes the topology defined by Hindy-Huang-Kreps, the topological dual $L^{\prime}$ is not a lattice, so that the Mas-Colell-Richard theorem does not apply and the equilibrium existence problem for an $e$-proper exchange economy $\mathcal{E}=\left(X_{i}, P_{i}, e_{i}\right)_{i \in I}$ with a total initial endowment $e=\sum_{i \in I} e_{i}>0$ remains an open issue, solved by

\footnotetext{
${ }^{11}$ The inductive limit topology generated on a vector space $M$ by the family of all its finite dimensional vector subspaces is the finest locally convex topology $\xi_{M}$ such that for each finite dimensional vector subspace $F$ equipped with its Euclidean topology the natural embedding $i: F \hookrightarrow\left(M, \xi_{M}\right)$ is continuous.

${ }^{12}$ The convex cone $K_{n}$ is an extremal subset of the convex cone $K_{n+1}$ if and only if $x=y+z, x \in K_{n}$ and $y, z \in K_{n+1}$ imply that $y, z \in K_{n}$.
} 
Bank-Riedel [17] under special assumptions on the utility functions representing the preferences of consumers.

If preferences are represented by utility functions, using Theorem 3.1 of Allouch-Florenzano [12], one can show that $\mathcal{E}$ has Edgeworth equilibria. Starting with an Edgeworth equilibrium allocation $x=\left(x_{1}, \ldots, x_{m}\right)$, and recalling that $L$ has the decomposition property but that $L^{\prime}$ is not a vector lattice in the order dual $L^{\sim}$, our proposition 5.1 shows that the $e$-proper economy $\mathcal{E}$ has a nontrivial quasiequilibrium $(x, p)$ whose price does not necessarily belong to $L^{\prime}$.

\section{REFERENCES}

[1] Aliprantis, C.D., Border K. C., 1999. Infinite Dimensional Analysis: A Hitchhiker's Guide. 2nd. ed., SpringerVerlag, Heidelberg and New York.

[2] Aliprantis, C.D., Brown, D.J., Burkinshaw, O., 1987. Edgeworth equilibria. Econometrica 55, 1109-1137.

[3] Aliprantis, C.D., Brown, D.J., Burkinshaw, O., 1987. Existence and Optimality of Competitive Equilibria, Springer-Verlag, Heidelberg and New York, 1990.

[4] C. D. Aliprantis, D. J. Brown, I. A. Polyrakis, and J. Werner, Portfolio dominance and optimality in infinite security markets, J. Math. Econom. 30 (1998), 347-366.

5] C. D. Aliprantis and O. Burkinshaw, Locally Solid Riesz Spaces, Academic Press, New York and London, 1978.

[6] C. D. Aliprantis and O. Burkinshaw, Positive Operators, Academic Press, New York and London, 1985.

[7] C. D. Aliprantis, M. Florenzano, F. Martins Da Rocha and R. Tourky, General equilibrium in infinite security markets, J. Math. Econom., forthcoming

[8] C. D. Aliprantis, P. K. Monteiro, and R. Tourky, Non-marketed options, non-existence of equilibria, and nonlinear prices, J. Econom. Theory, forthcoming.

[9] C. D. Aliprantis and R. Tourky, The super order dual of an ordered vector space and the Riesz-Kantorovich formula, Tran. Amer. Math. Soc. 354 (2002), 2055-2077.

[10] C. D. Aliprantis, R. Tourky, and N. C. Yannelis, The Riesz-Kantorovich formula and general equilibrium theory, J. Math. Econom. 34 (2000), 55-76.

[11] C. D. Aliprantis, R. Tourky, and N. C. Yannelis, A theory of value: equilibrium analysis beyond vector lattices, J. Econom. Theory 100 (2001), 22-72.

[12] N. Allouch and M. Florenzano, Edgeworth and Walras equilibria of an arbitrage-free exchange economy, Economic Theory, forthcoming.

[13] T. Andô, On fundamental properties of a Banach space with a cone, Pacific J. Math. 12 (1962), 1163-1169.

[14] A. Araujo and P. K. Monteiro, Equilibrium without uniform conditions, J. Econom. Theory 48 (1989), 416427.

[15] J. P. Aubin, Mathematical Methods of Game and Economic Theory, North-Holland, Amsterdam and New York, 1979.

[16] V. Barbu and T. Precupanu, Convexity and Optimization in Banach spaces, D. Reidel Publishing Company, Boston and Lancaster, 1986.

[17] P. Bank and F. Riedel, Existence and structure of stochastic equilibria with intertemporal substitution, Finance and Stochastics, forthcoming.

[18] G. Debreu, Valuation equilibrium and Pareto optimum, Proc. Nat. Acad. Sci. U.S.A. 40 (1954), 588-592.

[19] G. Debreu and H. Scarf, A limit theorem on the core of an economy, Internat.l Econom. Rev. 4 (1963), 235-246.

[20] M. Deghdak and M. Florenzano, Decentralizing Edgeworth equilibria in economies with many commodities, Economic Theory 14 (1999), 297-310.

[21] K. Fan, I. L. Glicksberg, and A. J. Hoffman, Systems of inequalities involving convex functions, Proc. Amer. Math. Soc. 13 (1957), 617-622.

[22] M. Florenzano, Edgeworth equilibria, fuzzy core and equilibria of a production economy without ordered preferences, J. Math. Anal. Appl. 153 (1990), 18-36.

[23] M. Florenzano, C. Le Van, Finite Dimensional Convexity and Optimization, Studies in Economic Theory \#13 Springer-Verlag, Heidelberg and New York, 2001. 
[24] M. Florenzano and V. Marakulin, Production equilibria in vector lattices, Economic Theory 17 (2001), 577-598.

[25] A. Hindy and C. Huang, Intertemporal preferences for uncertain consumption: a continuous time approach, Econometrica 60 (1992), 781-801.

[26] A. Hindy, C. Huang and D. Kreps, On intertemporal preferences in continuous time, J. Math. Econom. 21 (1992), 401-440.

[27] P. J. Laurent, Approximation et Optimisation, Herman, Paris, 1972.

[28] A. Mas-Colell, The price equilibrium existence problem in topological vector lattices, Econometrica 54 (1986), 1039-1055.

[29] A. Mas-Colell, Valuation equilibria and Pareto optimum revisited, in W. Hildenbrand and A. Mas-Colell (eds.), Contributions to Mathematical Economics. In honor of Gérard Debreu, North-Holland, Amsterdam, 317-331, 1986.

[30] A. Mas-Colell and S. F. Richard, A new approach to the existence of equilibria in vector lattices, J. Econom. Theory 53 (1991), 1-11.

[31] P. K. Monteiro and R. Tourky, Mas-Colell's price equilibrium existence problem: the case of smooth disposal, University of Melbourne, 2002, Mimeo.

[32] J. J. Moreau, Fonctionnelles convexes, Séminaire sur les équations aux dérivées partielles, Collège de France, $1966-1967$.

[33] I. Namioka, Partially Ordered Linear Topological Spaces, Mem. Amer. Math. Soc., 24, Providence, RI, 1957.

[34] A. L. Peressini, Ordered Topological Vector Spaces, Harper \& Row, New York and London, 1967.

[35] K. Podczeck, Equilibria in vector lattices without ordered preferences or uniform properness, J. Math. Econom. 25 (1996), 465-485.

[36] R. T. Rockafellar, Convex Analysis, Princeton University Press, Princeton, NJ, 1972.

[37] R. T. Rockafellar and R. J.-B. Wets, Variational Analysis, Springer-Verlag, Heildelberg and New York, 1998.

[38] H. H. Schaefer, Topological Vector Spaces, Springer Verlag, New York and Heildelberg, 1971.

[39] R. Tourky, A new approach to the limit theorem on the core of an economy in vector lattices, J. Econom. Theory 78 (1998), 321-328.

[40] N. C. Yannelis and W. R. Zame, Equilibria in Banach lattices without ordered preferences, J. Math. Econom. 15 (1986), 85-110. 